\title{
MILANO CITTÀ DI ESPOSIZIONI
}

\author{
ENRICO DECLEVA (*)
}

SunTO. - Milano è la città italiana che, a partire dagli anni successivi all'Unità, ha organizzato con continuità il maggior numero di esposizioni, mostre, fiere, rassegne di settori produttivi e di attività le più varie. Tanta insistenza, pur nella diversità delle situazioni, è indicativa della funzionalità e del successo della formula. L'intervento si sofferma in particolare sull'Esposizione del 1881, primo momento di affermazione e di autorappresentazione della città produttiva, orgogliosa delle sue capacità realizzatrici, bene amministrata, in grado di cimentarsi con i ritmi di sviluppo e di trasformazione propri della modernità, senza tuttavia rompere i ponti con la tradizione e senza dimenticare, sul versante benefico e umanitario, i più deboli e i meno fortunati. Prima che la guerra europea modificasse irreparabilmente il quadro di riferimento, l'Esposizione internazionale del $1906 \mathrm{fu}$ un secondo momento centrale con il quale valorizzare il cammino percorso e i risultati raggiunti.

$$
* * *
$$

ABSTRACT. - Milan is the Italian city that, from the years following Italian unification onwards, has continually organized the highest number of expositions, exhibitions, trade fairs and reviews of productive sectors, in addition to the most varied kinds of activities. Such indefatigability, even in the most diverse of situations, is indicative of how well its formula has functioned. This paper is mainly about the Universal Exposition of 1881, the city's first opportunity to present its achievements and to project a self-image as a productive, well-administered city that was proud of its ability to get things done, and capable of keeping pace with the rhythms of development and transformation that went along with modernity. Yet Milan could also be a modern city without destroying its ties with tradition and without forgetting - in terms of social welfare and humanitarianism - the weakest and least fortunate. Before the War in Europe irreparably changed its frame of reference, the International Exposition of 1906 was the second central event through which the city could look back with satisfaction on the route it had taken and the results it had achieved.

(*) Istituto Lombardo, Università degli Studi di Milano, Italia.

E-mail: e.decleva@gmail.com 
1. Le sette esposizioni organizzate tra il 1871 e il 1906; le decine di fiere campionarie che si sono succedute dal 1920; le esposizioni di arte decorativa, prima all'Umanitaria, poi a Monza e infine presso la Triennale; le numerose mostre e rassegne a tema organizzate nel recinto della Fiera (basti solo pensare a quelle di valenza davvero strategica dedicate alle macchine utensili); i saloni (come quello del mobile, che ormai invade anche la città, o quello del ciclo e del motociclo); le sfilate delle case di moda, fino a giungere a quello che è per ora il punto di arrivo, Expo 2015: davvero non è un problema giustificare il titolo di questo convegno e del volume che ne raccoglie i materiali.

Credo che lo si possa sostenere senza tema di smentite. Fa parte dei connotati più specifici della Milano degli ultimi centoquarant'anni - se non fosse banale dirlo, del suo DNA - essere stata ed essere una "città di esposizioni". Che ha fatto cioè e fa di queste ultime una sorta di manifestazione normale e reiterata nel corso del tempo del suo modo di essere e di caratterizzarsi rispetto al resto del Paese e al mondo. Contando sui ritorni di vario tipo che ne conseguono, sulla loro forza di richiamo, sulle reti di relazioni che permettono di sviluppare e coltivare.

Se il fenomeno è evidente, se evocarlo può servire a una migliore comprensione della fisionomia e delle caratteristiche della città, è anche indubbio che esso ha preso le mosse da una esperienza iniziale che non ha mancato di condizionare il poi. Se non altro perché ha avuto successo e ha incentivato la sua riproposizione nel tentativo di rinnovarlo o in funzione di altre esigenze maturate nel frattempo.

Non mi riferisco tanto all'ancora approssimativa esposizione del 1871, comunque degna di nota per essersi fregiata per la prima volta del titolo di "industriale"; o a quella "storica di arte industriale" del 1874 (che pure ha avuto un suo significato). Il punto di riferimento obbligato per una riflessione sul tema è la ben più importante rassegna realizzata nel 1881, rilevante in sé, per le sue caratteristiche e per i suoi effetti, e nella valenza simbolica che ha finito con l'assumere. Vero e proprio evento nella storia milanese di quei decenni, e da vari punti di vista. E non a caso posta quale primo termine di richiamo nel titolo stesso del convegno.

2. Che a lanciare l'idea di fare a Milano qualcosa di simile a quanto era stato da poco realizzato a Parigi con l'Esposizione del 1878 sia stato il proprietario d'uno dei più importanti alberghi cittadini dell'epoca indica con immediatezza che cosa, in primo luogo, ci si proponesse di fare: richiamare a Milano visitatori e curiosi, con i vantaggi che ne sarebbero 
derivati. Ma dal momento in cui il progetto prese più concreta forma, anche le sue motivazioni si fecero più specifiche e, in certo senso, meno contingenti. Come metteva in evidenza il Manifesto steso per l'occasione:

Tenere il lavoro in onore, rimuovere gli ostacoli, sorreggere l'industria con mano amica, e fare in modo che sia circondata da un influsso fecondo che la spinga sulla via del progresso e della perfezione, ecco, in riassunto, il movente che ci deve guidare e il fine a cui tendere.

"Lavoro", "industria", "progresso": i termini di riferimento erano dunque questi. Ma fu soprattutto grazie all'arrivo tra gli organizzatori di Giuseppe Colombo, titolare di Meccanica industriale all'Istituto tecnico superiore - la scuola politecnica avviata meno di vent'anni prima da Francesco Brioschi - che la rassegna espositiva assunse la sua valenza più tipica. Fu infatti opera precipua di Colombo e dei suoi allievi Saldini e Ponzio progettare e configurare quella Galleria delle macchine e del lavoro in cui per la prima volta i visitatori poterono vedere anche in Italia apparecchiature e macchinari in movimento. Dando così una più specifica valenza allo stesso termine "industria", sin lì sinonimo di qualsiasi tipo di attività e intraprendenza e da allora più strettamente e specificamente associato all'uso di macchine. Fu ancora Colombo a occuparsi della collocazione di queste ultime, assumendo più in generale una mole ingentissima di lavoro e di impegni, quasi a dimostrazione, che più eloquente forse non avrebbe potuto essere, di quanto rilevanti ed efficaci, nel risolvere le situazioni e superare le difficoltà, potessero essere le competenze di un ingegnere: e chi più ingegnere di lui, autore di quel fortunatissimo Manuale, viatico per decenni, nelle successive edizioni, degli adepti alla professione? E' degno di nota, a proposito dell'impegno e delle fatiche affrontate, che quando, sei anni dopo, Saldini, principale organizzatore dell'Esposizione di apparecchi per la macinazione e la panificazione che si svolse nel 1887, chiese a Colombo di entrare nel Comitato esecutivo, questi gli rispondesse di essere in realtà "saturo di Esposizioni", e di accettare bensì, ma a condizione "di non aver da fare più di quanto richiedono le Commissioni ordinarie, e in ogni modo non come io e Lei e Ponzio ne ebbimo nel 1881" .

1 G. Colombo a C. Saldini, 15 febbraio 1886, in Archivio storico civico, Milano, Fondo Esposizioni e Fiere, 1887, cart. 1. fasc. Comitato esecutivo e promotore. 
Principale regista nella predisposizione della parte più significativa dell'Esposizione del 1881, Colombo ne era poi stato in certo senso il principale esegeta, avendo avuto modo di misurare in tutta la loro entità i miglioramenti intervenuti rispetto all'Esposizione italiana agraria, industriale e artistica (come la si era titolata) organizzata a Firenze nel 1861, da lui fatta allora oggetto dalle colonne de "La perseveranza" di una analisi lucida e realistica, in netta controtendenza rispetto ai trionfalismi di circostanza. E mettendo per contro bene in evidenza quanto si dovesse ancora "apprendere" prima di potersi davvero affrancare dalla dipendenza dall'industria estera. Proprio questo era accaduto. L'apprendistato c'era stato e se ne potevano vedere i risultati. Il paese aveva inequivocabilmente "mostrato cosa poteva e sapeva fare nel campo industriale", e in qualche settore come quello di sua competenza delle "industrie delle macchine" - più che in altri. Con esiti sostanziali.

A conferma che non si era sbagliato a scegliere quella strada e a porre Milano, la Milano industriale descritta sempre da Colombo in una delle pubblicazioni uscite per l'occasione, al centro delle dinamiche in atto. Dinamiche che naturalmente non si concludevano con quel passaggio: e tantomeno con riguardo alle attività e alle iniziative di Colombo, che anzi assunsero, proprio dopo il 1881, una connotazione nuova, legata allo sviluppo dell'industria elettrica, con tutti gli effetti che questa avrà anche sulla configurazione della Milano industriale.

Ma l'Esposizione del 1881 aveva dato anche altre dimostrazioni. Anche se i torinesi trovarono di che ridire e si impegnarono a far meglio, attivando di lì a tre anni una loro Esposizione intorno al castello e al parco del Valentino, la rassegna milanese del $1881 \mathrm{fu}$ anche un'importante prova organizzativa superata brillantemente, e con risultati economici che sarebbero risultati positivi anche prescindendo dal contributo governativo, d'altronde contenuto. Già lo si era registrato, ma su una scala molto più modesta nel 1871. Ora lo si poteva ribadire con più forza. Milano era in grado di fare da sé, potendo contare sulla solidità delle sue strutture amministrative e di servizio e sull'impegno e l'amore civico dei suoi abitanti, sul loro spirito di concordia, che non escludeva una persistente conflittualità, ma che era poi pronto a scattare e a superare i contrasti quando se ne presentavano le ragioni.

E le numerose iniziative editoriali realizzate per l'occasione, a loro volta emblema di una particolarità cittadina - la rilevanza cioè delle 
pubblicazioni librarie e giornalistiche, altrove prerogativa della città capitale - consolidavano e rafforzavano l'immagine della "capitale morale", identificandone e celebrandone i pregi e i punti di forza.

3. Molto del seguito, e delle fortune della formula espositiva in una città come Milano, è identificabile nel particolare impasto di elementi emersi in quella circostanza così fortunata. E, in primo luogo la scelta di fondo, di configurare la città e quantomeno una parte della regione in senso industriale e produttivo, legando a questo nuovo destino, e alla capacità di essere parte attiva nei processi di modernizzazione in atto, il ruolo e il peso di Milano nella compagine nazionale. L'esposizione del 1881 aveva dimostrato che lo stereotipo dell'Italia solamente agricola non teneva conto di un'altra Italia, un'Italia industriale sia pure non presente in tutte le regioni, che per contro esisteva e riteneva di avere tutte le ragioni per affermarsi e per chiedere di essere sostenuta in questo anche da più adeguate politiche doganali.

Era dunque in certo senso implicito in quel primo episodio che ci fosse un poi, che ne seguissero altri. Sempre nella presunzione - in realtà tutta da verificare e rispetto alla quale non mancheranno di farsi sentire crescenti dubbi e preoccupazioni - che si potesse contare sulla particolare configurazione della città e sulle caratteristiche del suo modello di industrializzazione per evitare i contraccolpi sociali verificatisi altrove, là dove si erano determinate rilevanti concentrazioni operaie.

L'appello agli imprenditori delle altre regioni perché venissero qui a dare la dimostrazione di quello che erano capaci di fare, non faceva d'altra parte dimenticare che una parte consistente degli espositori era milanese e lombarda e che erano localizzati in primo luogo qui i fattori trainanti dell'intero processo.

Ne derivava, già nel 1881 ma poi con continuità nelle tante rassegne espositive milanesi anche internazionali che sarebbero seguite, una sorta di doppia prospettiva, che collegava e metteva a confronto il livello e il grado di concorrenzialità della produzione locale ai livelli raggiunti altrove, su scala semplicemente nazionale dapprima, internazionale poi. Dove era in ogni caso sostanziale la scelta di fondo, di cimentarsi per quanto possibile alla pari con i ritmi di sviluppo e di trasformazione propri della modernità, dotandosi degli strumenti per affrontarla, ponendosi in tal modo al centro di uno snodo di relazioni certo favorite dalla geografia e dalla rete dei trasporti. Non è un caso 
che l'età d'oro delle esposizioni milanesi sia compresa nell'arco di tempo che va dal traforo del San Gottardo all'apertura del valico del Sempione, che avevano collegato Milano prima verso la Svizzera e la Germania, poi verso la Francia, facendone il luogo di raccordo sia di ciò che veniva sia di ciò che andava in quelle due direzioni: merci, capitali, uomini, idee.

E non era di piccolo significato l'altra indicazione, cui ho già accennato, legata all'esperienza del 1881: e cioè la pronta rispondenza della città o dei suoi settori più significativi a sostenere lo sforzo necessario per la realizzazione della manifestazione, identificandosi nell'obiettivo di farle riuscire, avendo le competenze e le capacità per organizzarla al meglio, richiamando abbastanza visitatori, milanesi e non, per far tornare i conti, senza bisogno del ricorso a fondi governativi, o limitandolo al minimo.

Così come non era destinato a rimanere un episodio la valorizzazione allora compiuta della città, del suo particolare impasto di tradizione e modernità, della sua intraprendenza, del livello delle sue strutture, della sua forte connotazione in senso tecnico, che non doveva però significare l'assunzione di quella sola dimensione di riferimento, secondo l'ammonimento rilanciato proprio nei giorni dell'Esposizione dal vecchio Cesare Correnti affinché la città si facesse bensì "officina di sapienti industrie", ma restando "il santuario delle arti belle" e "un ateneo di buoni studi". Pena, altrimenti, non rimanendo "la più civile", di diventare "presto la più villana delle città" 2 .

A coltivare i buoni studi, entro la città industriale che pure si imponeva con le sue esigenze e con i suoi connotati più specifici, provvedeva in effetti, pur con tutti i suoi limiti e la modestia del numero degli iscritti, l'Accademia scientifico-letteraria, i cui due corsi di laurea in Lettere e in Filosofia vennero proprio allora affiancati da una Scuola di lingue e letterature moderne, resa accessibile anche alle giovani uscite dalla civica Scuola superiore femminile intitolata a Manzoni, promossa da Carlo Tenca. Prendendo un paio d'anni prima le difese proprio dell'Accademia scientifico-letteraria era stato del resto il "Corriere della sera", il nuovo quotidiano fondato da Eugenio Torelli Viollier, destinato ad affermarsi come l'organo di stampa più moderno e diffuso del Paese, a sostenere con decisione che la forza d'influenza e il prestigio

2 C. Correnti, Palinodia, in Milano e i suoi dintorni, Milano, Civelli, 1881, 311. 
d'una città non dipendevano che parzialmente "dalla sua operosità industriale e commerciale e dalla sua prosperità materiale". L'elemento distintivo era semmai costituito dal suo "valore intellettuale":

Bordeaux, Marsiglia, Rouen, malgrado le loro grandi ricchezze, hanno poca influenza sull'indirizzo politico della Francia, perché non sono che ricche. Il lustro di Milano sarebbe dimezzato se gli togliessero i suoi editori, i suoi giornali, le sue svariate pubblicazioni, per le quali gran parte d'Italia si rifornisce da noi d'alimento mentale. Questo fenomeno di studi e di produzione d'ingegno, bisogna mantenerlo3.

E non si può dire, guardando le cose in prospettiva, che sforzi e sviluppi in quel senso non si siano manifestati negli anni e nei decenni a venire e che Milano non sia riuscita a connotarsi anche in quel senso: centro indiscusso, senza nulla togliere alla rilevanza di imprese editoriali operanti altrove, di quella che si è poi chiamata, anche fra noi, con una accezione più o meno positiva, l'industria culturale.

4. Nessuna delle rassegne espositive che seguirono ebbe la valenza progettuale e le caratteristiche di complessiva omogeneità proprie di quella del 1881. L'autorappresentazione della città, dei suoi interessi e delle sue aspirazioni, venne da allora in poi affidata semmai all'accostamento di distinti segmenti espositivi, espressione, ciascuno, di uno specifico retroterra: ma anche della volontà di tenere il passo con le novità prodotte dalla modernità avanzante e di mostrarne i pregi. Si trattasse dell'illuminazione elettrica, elemento di particolare attrazione della già citata esposizione del 1887; o di alcune delle mostre che composero le Esposizioni riunite del 1894: Sport, Fotografia, Pubblicità. O di una parte di quelle che vennero aggregate all'Esposizione internazionale del 1906, imperniata sul tema dei trasporti: non solo quelli ferroviari, come si conveniva all'occasione, legata all'apertura del valico del Sempione, ma anche l'automobilismo, il ciclismo, l'aeronautica.

E non era tutto. Nella periodica raffigurazione che Milano diede di sé nell'età liberale, non mancò mai uno spazio dedicato al versante benefico e umanitario, alle preoccupazioni per i bisognosi, per l'infanzia, per i meno fortunati, per le istituzioni create a loro sollievo o per rilevarne le

3 “Corriere della sera", 15-16 maggio 1879, Calibano a Milano. 
sorti. Mi limito a ricordare in questa sede quello che è stato in certo senso il punto d'arrivo d'una attenzione siffatta: il padiglione della Società Umanitaria nell'Esposizione del 1906. Dove si esprimeva il presupposto di fondo di quella singolare istituzione, che per affrontare i mali della società - $\mathrm{i}$ retaggi del passato così come $\mathrm{i}$ traumi dell'incipiente industrialismo - occorresse puntare non sul conflitto ma sui benefici di un etica sociale fondata sulla valenza emancipatrice del lavoro e sulla migliore qualità della vita che esso poteva produrre e al tempo stesso incentivare. Nasceva anche da qui la particolare attenzione tenuta viva dall'Umanitaria per le arti decorative, presentate come elemento gratificante anche della casa operaia. Le arti in questione erano d'altronde un tema centrale, più in generale, dell'Esposizione del 1906, essendo già state presenti in alcune di quelle che l'avevano preceduta, a cominciare da quella, già ricordata nella sua specificità, del 1874. Ed è appena il caso di rilevare gli elementi di continuità che legano l'arte industriale di quei decenni, nei passaggi che la caratterizzarono, all'affermazione della città nel campo del design in tempi a noi più vicini.

Ma c'è stata anche un'altra Milano che si è in certo senso espressa o si è sentita rappresentata nelle sue prime esposizioni: la Milano "gaudente" e "gaia" , pronta a rispondere con solerzia alle sollecitazioni di cui era fatta oggetto, e ad affollare quindi di volta in volta i padiglioni espositivi e, forse ancor più, gli spazi di richiamo e di attrazione tutt'intorno, d'altronde concepiti e realizzati proprio con quello scopo. Con tutte le valenze prospettiche che se ne vogliano trarre: le dimensioni dirompenti e di massa dei fenomeni consumistici o legati al tempo libero dei nostri giorni non devono far dimenticare le loro tanto più contenute, ma non meno significative prime manifestazioni intervenute in altre epoche, quando quei processi, derivanti anch'essi dalla modernità avanzante, hanno in definitiva preso avvio: magari destando l'allarme preoccupato di chi vi vedeva una deviazione impropria rispetto alla natura, che si sarebbe voluto solo severamente didascalica, delle esposizioni.

5. Se l'Esposizione del 1881 risulta più che mai rappresentativa dell'avvio del processo di affermazione della città, superata la fase difficile della ricerca di un ruolo dopo l'Unità, quella del 1906 può essere

4 Cfr. Il Ventre di Milano. Fisiologia della Capitale morale per cura di una società di letterati, Milano, Aliprandi, 1888, I, 8. 
legittimamente considerata un punto d'arrivo, emblema ed espressione di una Milano orgogliosa del cammino percorso e dei progressi realizzati, urbanisticamente rinnovata dal piano regolatore elaborato negli anni '80, dotata o in corso di dotarsi di strutture e servizi in linea con quanto di più avanzato esisteva in Europa, apparentemente in grado non solo di sviluppare, ma anche di controllare e orientare al meglio, nella dimensione politico-amministrativa e sociale, gli elementi di modernità legati al suo sviluppo in senso industriale, commerciale, finanziario. La giunta guidata dal sindaco Ponti, prosecutrice, pur con i suoi connotati moderati, di molti progetti impostati dalla giunta precedente, espressione della vittoria dei partiti popolari dopo la crisi del 1898 , poteva apparire la risposta più adeguata, sul piano amministrativo, rispetto all'insieme di esigenze che ne derivavano.

Ma non era propriamente così, come si rileverà in alcuni interventi successivi. L'Esposizione del 1906 si colloca in realtà allo spartiacque tra due diverse fasi anche della vita cittadina, politica e sociale. Per non dire di quello che seguirà, tra la settimana rossa e lo scoppio della prima guerra mondiale. E, poi nel primo dopoguerra.

Espressione quanto mai indicativa, in versione ambrosiana, delle certezze e delle illusioni, riferite sia al quadro interno sia a quello internazionale, di quella che col senno di poi si è chiamata la Belle Époque, la sequela di esposizioni cui si è accennato non poteva in ogni caso non esaurirsi, in quelle particolari forme, col tragico venir meno di quelle illusioni e di quelle presunte certezze nel 1914.

Una parte almeno delle esigenze che avevano trovato l'espressione alla quale si è accennato nella precedente stagione sarebbero riemerse e avrebbero trovato ampio spazio anche nei nuovi tempi, nonostante alcuni dei connotati che furono loro propri. Chiuso il ciclo delle esposizioni di vecchio modello - quali ne fossero le eredità e i lasciti effettivi - sarebbe toccato a quel punto alla Fiera campionaria e alla Triennale, realtà ognuna ben specifica e con funzioni proprie, continuare a trasmettere a Milano, con l'evidenza di ciò che erano in grado di proporre, al di là dei filtri cui dovevano sottostare, i messaggi e le suggestioni, di diverso segno, della modernità. E dimostrare nello stesso tempo che Milano, nella sua specificità, continuava ad esserne parte integrante e attiva, centro e snodo di una rete destinata a svilupparsi ulteriormente nel corso del tempo, secondo il destino proprio dei grandi centri metropolitani. Con le dinamiche e i problemi loro propri, ai quali, anche se si vorrebbe, non è dato sfuggire. 
E che in effetti, volenti o nolenti, abbiamo di fronte anche oggi, se appena cerchiamo di capire o di immaginare che cosa implichi essere davvero una città all'altezza di una iniziativa delle dimensioni e della rilevanza di Expo 2015. Beninteso: al di là delle frasi fatte e delle fughe in avanti. 